\title{
Philonsorbonne
}

$10 \mid 2016$

Année 2015-2016

\section{Doctorales 2015}

Résumés des Doctorales de Philosophie (2015)

\section{OpenEdition}

\section{Journals}

Édition électronique

URL : https://journals.openedition.org/philonsorbonne/809

DOI : 10.4000/philonsorbonne.809

ISSN : 2270-7336

Éditeur

Publications de la Sorbonne

Édition imprimée

Date de publication : 1 janvier 2016

Pagination : 89-129

ISSN : 1255-183X

\section{Référence électronique}

«Doctorales 2015 », Philonsorbonne [En ligne], 10 | 2016, mis en ligne le 19 janvier 2016, consulté le 08 juin 2021. URL : http://journals.openedition.org/philonsorbonne/809; DOI : https://doi.org/10.4000/ philonsorbonne.809

(c) Tous droits réservés 


\section{Doctorales 2015 *}

\section{ÉPISTÉMOLOGIE HISTORIQUE : TRADITION ET MÉTHODE}

Qu'est-ce que l'épistémologie historique?

Un aperçu historico-méthodologique à partir de bachelard

Matteo VAGELLI

Une thérapie politique à partir de Canguilhem

Gabriel VISSIO

Remarques sur le rapprochement entre Bergson, Canguilhem et le vitalisme Marcos CAMOLEZI

La normativité du vivant et la normativité des sciences chez Canguilhem

Ivan MOYA DIEZ

Canguilhem au Brésil :

comment on écrit l'histoire épistémologique de la santé publique

Tiago AlMEIDA

Between Objectivity and History:

An Analysis of Ian Hacking's Styles of scientific Reasoning

Wenbo LIANG

\section{PHILOSOPHIE DES MATHÉMATIQUES ET DU LANGAGE}

Représentations et symbolismes en mathématiques

David WASZEK

L'austérité du langage dans Le rapport de Brodie de Borgès

Florencia DI ROCCO

\section{MÉCANISMES PSYCHOLOGIQUES ET PHILOSOPHIE}

Les processus inconscients en jeu dans la créativité et la prise de décision

Claude MAYER

La récapitulation ontophylogénétique en psychologie

Sylvain BOSSELET

Causalité et philosophie de l'esprit

Marzia MichELIZZA

La dynamique des pensées sur soi-même

Pedro Henrique MuNIZ

(*). Dans cette rubrique sont publiés les résumés des interventions ayant eu lieu lors des Doctorales de Philosophie organisées à la Sorbonne les 03, 04, 05 et 06 juin 2015, par les doctorants Audrey Benoit (CHSPM), Pierre DE JouvenCOURT (CETCOPRA), Daniel LARose (Gramata), Yoann Malinge (PhiCo) et Delphine Olivier (IHPST). 


\section{LES FORMES DU MODĖLE}

La réification en expérimentation animale : nécessité ou convention?

Jacques CABARET

Analoque naturel et transition énergétique

Dorine MONTOUT

La reconstruction ingénieuse du modèle chez Baltasar Gracián

Thibault BARRIER

Le modèle et la norme dans le Discours

philosophique de la modernité d'habermas

Clotilde NOUËT

Instituer et destituer des modèles. La précarité des alliances chez Nietzsche

Arnaud SOROSINA

\section{PHILOSOPHIE SOCIALE ET POLITIQUE}

Démocratiser le travail.

Ce que nous apprenent les expériences autogestionnaires

Camille TERNIER

La théologie politique et l'histoire des concepts :

le cas de Carl Schmitt, à travers Hans Blumenberg et Reinhart Koselleck

Masoud SINAEIAN

Que critique Adam Ferguson dans la division du travail?

Sonia BOUSSANGE-ANDREI

\section{AUTOUR DE LA GUERRE : REGARDS CROISÉS}

Les causes des guerres civiles et étrangères chez Platon

Mamadou SOUMARÉ

La guerre pour les sujets d'un autre chez grotius.

Du droit de punir à la permission d'intervenir

Lyess BOUDERBALA

Le rôle des intellectuels dans les transformations politiques et sociales de la société kurde après l'apparition de l'opposition en 2009

Shawnm Yahya KIDIR

Une militarisation des savoirs :

de l'usage stratégique des sciences humaines durant la guerre d'Algérie

Théophile LAVAULT

\section{PERSISTANCE ET INDIVIDUALITÉ DES ÊTRES VIVANTS}

La stabilité des vivants :

comparaison des régimes d'auto-maintien des organismes et des écosystèmes 133 Victor LEFÈVRE 
La robustesse des organismes comme critère d'individualité

Anna Maria DIELI

Complexité et phase de constitution des corps chez Spinoza

Vincent LEGEAY

La distinction entre action parfaite

et mouvement inachevé en Métaphysique @-6

Jean-Pierre VANANDRUEL

La place de l'organisme dans les dynamiques évolutives :

Persistance chagement, sélection

Nicola BERTOLDI

\section{PHILOSOPHIES FÉMINISTES}

Autour de l'épistémologie féministe

Mona GÉRARDIN-LAVERGE

\section{PHILOSOPHIE MORALE ET AGENTIVITÉ}

Hume et la critique de la souveraineté pratique de la raison

Sophie BERGONT

Les faits et les raisons, une dichotomie?

Sylvain THEULLE

PHILOSOPHIE DE LA PERCEPTION, ONTOLOGIE ET ESTHÉTIQUE

La distinction entre la perception et la sensation dans le toucher

Alireza BANI SADR

L'œil et l'esprit chez Spinoza : la peinture est-elle toujours muette ?

Nicolas BOUTELOUP

Le rêve et la vie : éléments d'une ontologie du surréalisme

Louis MORELLE

\section{SUJET ET RÉALITÉ}

Repenser le réalisme :

quelques contributions pragmatistes (Dewey et Putnam)

Aurora ALBAN

Être et image : une approche de la subjectivité chez maître Eckhart

Pierre-Luc DESJARDINS

Inhérence, immanence, ipséité : Heidegger, lecteur des scolastiques 
Qu'est-ce qu'un champ?

Aspects ontologiques de l'électromagnétisme de Faraday

Kévin CAPPELLI 


\section{ÉPISTÉMOLOGIE HISTORIQUE : TRADITIONS ET MÉTHODES}

\section{Qu'est-ce que l'épistémologie historique ? Un aperçu historico- méthodologique à partir de Bachelard}

Dans mon intervention je me propose de donner quelques repères pour répondre à la question : "What (Good) is Historical Epistemology? », qui, depuis des années, semble hanter nombreux débats en histoire et philosophie des sciences. Je vise d'abord à fournir un cadre général aux thématiques et aux auteurs de ce type d'épistémologie, en traçant une brève histoire du terme "épistémologie historique » et cartographiant certains de ses différents usages. Je m'appuierai en particulier sur la pérennité de certains concepts de la boîte à outils bachelardienne dans la tradition de l'épistémologie historique. D'un côté, le tournant normatif imprimé par Bachelard à l'histoire des sciences à travers le principe de récurrence ( $L a$ formation de l'esprit scientifique, 1938 ; Le matérialisme rationnel, 1953) a, en un certain sens, rendu possibles les travaux de Canguilhem (Le normal et le pathologique, 1966) et de Foucault (Naissance de la clinique, 1963) sur l'histoire de la médecine. De l'autre, le rationalisme appliqué et la notion de phénoménotechnique (1953) ont anticipé certains des aspects plus novateurs de la philosophie du laboratoire de Ian Hacking (Representing and Intervening, 1983). À travers cette référence à Bachelard, je vise donc à faire interagir indirectement la tradition française et la tradition contemporaine, principalement anglo-saxonne, dite de l'« historical epistemology».

En marge de l'exposé, qui doit servir d'introduction aux autres interventions de l'atelier «Épistémologie historique: traditions et méthodes », je me propose également de présenter le projet lié au site web episthist.hypotheses.org, qui vise la création d'un réseau international des chercheur(se)s qui travaillent dans le domaine, en constante expansion, de l'épistémologie historique.

Matteo VAGELLI

[Doctorant en cotutelle (Université Paris I Panthéon-Sorbonne/Scuola Alti Studi Fondazione San Carlo) sous la direction du Professeur Jean-François Braunstein (PhiCoExeCo) et du Professeur Arnold I. Davidson (University of Chicago)]. 


\section{Une thérapie politique à partir de Canguilhem}

Nous sommes désormais habitués à l'idée qu'il y a des vraies «maladies sociales » ou même des «épidémies » (par exemple la crise comme quelque chose qui peut infecter les États). L'utilisation sociale et politique des concepts médicaux aujourd'hui a transmis au discours politique une certaine idée de thérapie « sociale » qui voit son but comme le rétablissement d'une santé originaire ou comme retour à une " grande époque ».

La première partie de cet exposé montre le processus de naturalisation de la santé opéré par la médecine du XIX ${ }^{\mathrm{e}}$ siècle. En particulier cette naturalisation de la santé est passée par la naturalisation de la maladie et la conception de la pathologie comme science d'un phénomène naturel qui peut être étudié par des pratiques de mensuration physique et chimique. La maladie, dans ce cadre, est considérée comme un phénomène naturel en tant que phénomène étudiable par les sciences de la nature, idée très forte dans l'école physiologique allemande. Si nous ajoutons à l'idée de la maladie comme phénomène mesurable la conception de l'état pathologique comme variation quantitative de l'état physiologique, c'est la santé aussi qui est naturalisée. C'est par ce double mouvement que le laboratoire de physiologie a dominé sur les niveaux clinique et thérapeutique de la médecine.

À ce cadre théorique correspond une pratique médicale qui donne un rôle dominant au moment diagnostique comme moment de mensuration objective et d'évaluation positive de la maladie. Le diagnostic a une double implication : (1) il est une évaluation individuelle de la maladie et (2) une mensuration statistique épidémiologique. La conséquence de cette double détermination est la corrélation du diagnostic et des pratiques de prévention : même les individus, même la population ont un "devoir à la santé » et doivent être évalués et soumis à des pratiques de prévention.

La même situation se vérifie dans la pratique sociale et politique, quand le lexique et les concepts médicaux sont assimilés dans ce champ de discours : il faut bien diagnostiquer la situation « pathologique » des États et annuler le risque de contamination, et il faut suivre un précis protocole thérapeutique pour rétablir la normalité et récupérer la "santé sociale ». Cet exposé veut démontrer que, dans la pensée de Georges Canguilhem, en particulier dans son idée de santé et de guérison, il y a les ressources nécessaires pour une nouvelle conception de thérapie sociale et pour une différente «métaphorologie » médicale pour la société.

Gabriele VISSIO

[Doctorant à l'Université de Turin (Italie). Il collabore régulièrement avec l'équipe de recherche du Ce.S.Pe.C./Centre d'Études sur la Pensée Contemporaine (Cuneo, Italie) et à la rédaction de la revue "Lessico di Etica pubblica " (www.eticapubblica.it). - Sujet de thèse : Georges Canguilhem. - Directeurs de thèse : Jean-François Braunstein (Paris I) et Graziano Lingua (Università di Torino). - Laboratoires de rattachement : PhiCo (EA3562)/Centre de philosophie contemporaine de la Sorbonne (Université Paris I), et DEF/ Dipartimento di Filosofia e Scienze dell'Educazione (Università di Torino]. 


\section{Remarques sur le rapprochement entre Bergson, Canguilhem et le vitalisme}

Le fait que Henri Bergson et Georges Canguilhem se rejoignent dans leur souhait de déceler une pensée originale du vivant, ainsi que les successives lectures, analyses et évaluations de l'un par l'autre, suggèrent une ressemblance plus ou moins grande de l'horizon philosophique que les deux philosophes pourraient se partager. Du côté bergsonien, on a affaire à un auteur polyvalent, défenseur d'une pensée "capable de suivre la réalité concrète dans toutes ses sinuosités » (La pensée et le mouvant, « La philosophie de Claude Bernard). Celle-ci serait édifiée sur la base du dynamisme psychique exprimant le plus nettement possible l'écart entre deux conceptions du temps distinctes et en quelque sorte opposées : celle $\mathrm{du}$ vivant (intuitive, psychique, organique, réelle) et celle de la matière (scientifique, mécanique, conventionnelle, illusoire). Du côté de Canguilhem, tout du moins de ses publications datant des années 1940, on a affaire à un philosophe attiré par la technique et par une conception de la médecine faisant comprendre la vie non comme le déploiement de règles fixes, mais comme une créativité (des outils aux normes) dans l'enjeu continu de l'organisme dans son milieu. Une suite de reprises explicatives ou appréciatives de l'héritage de Bergson par Canguilhem apparaît dans les enseignements des années 1940 à la Faculté de Lettres de Strasbourg ou dans les conférences de La connaissance de la vie, où l'estime de l'enseignant pour le « grand philosophe » n'est pas moindre. Il est également vrai qu'en raison de cela, l'accord supposé entre Bergson et Canguilhem saurait se traduire en une défense de la légitimité d'une pensée autonome du vivant, irréductible à l'explication physico-chimique, si bien que ces deux philosophes pourraient être associés à l'idée ou même à l'histoire du vitalisme. Il demeure pourtant que cette convergence paraît confrontée à des problèmes avant d'aboutir à une conclusion opportune. À partir d'une sélection de textes ne se résumant pas à ceux datant des années 1940, je souhaiterais donc émettre des remarques sur la portée des points de convergence et de divergence entre ces deux philosophes, en essayant de souligner l'hétérogénéité de leurs trajectoires.

Marcos CAMOLEZI

[Doctorant en Philosophie sous la direction de Jean-François Braunstein, Université Paris I/Panthéon-Sorbonne (PhiCo-ExeCO), et de Franklin Leopoldo e Silva, Universidade de São Paulo (PPGFil-USP). Boursier Fapesp, Brésil]. 


\section{La normativité du vivant et la normativité des sciences chez Canguilhem}

L'objectif de cette intervention est de comprendre l'appropriation de l'épistémologie bachelardienne par Canguilhem à l'égard des différents usages de la notion de normativité. Tout d'abord, dans sa célèbre thèse de 1943, Canguilhem fait une analyse historique du problème du normal et du pathologique. Il s'agit là, d'une part, de critiquer le réductionnisme et le déterminisme de certaines thèses médicales, en affirmant l'originalité de la vie et l'impossibilité de faire l'économie de jugements de valeurs dans la connaissance médicale et, d'autre part, de proposer une conception normative du vivant, à savoir, la normalité entendue comme normativité, capacité à surmonter des obstacles et à créer de nouvelles normes de vie dans un débat polarisé en valeurs avec le milieu.

Malgré l'approche historique de sa thèse, ce n'est que quelques années plus tard que Canguilhem commence son œuvre d'historien des sciences, après sa rencontre avec Gaston Bachelard et son "épistémologie historique ». Cette dernière approche propose une histoire normative et récurrente des sciences, sous le modèle d'un tribunal qui porte des jugements de valeurs sur le savoir du passé d'après le présent, autrement dit, une histoire jugée et sanctionnée par le savoir du temps présent.

À la différence de Bachelard dont l'œuvre porte essentiellement sur la physique, les études d'histoire des sciences de la vie de Canguilhem obligent à s'interroger sur les rapports entre la connaissance et la vie. En effet, dans l'histoire de la formation des concepts de sciences du vivant, on ne peut pas ignorer que c'est le vivant humain lui-même qui formule des concepts en surmontant des erreurs et des obstacles. L'épistémologie historique de Canguilhem constitue ainsi à la fois une histoire normative des sciences et, en utilisant les mots de Foucault, une recherche de la normativité interne aux différentes activités scientifiques.

Ivan MOYA-DIEZ

[Doctorant à l'Université Paris I/Panthéon-Sorbonne, rattaché au Centre de Philosophie Contemporaine de la Sorbonne].

\section{Canguilhem au Brésil : comment on écrit l'histoire épistémologique de la santé publique}

L'idée que l'historicité des notions de santé et de maladie a une influence directe sur la pratique médicale est loin d'être une inquiétante nouvelle pour les historiens brésiliens - au moins depuis la réception au Brésil de la pensée de Georges Canguilhem, avec la thèse doctorale « $\mathrm{O}$ dilema preventivista: contribuição para a compreensão e crítica da Medicina 
Preventiva ", présentée par Sérgio Arouca à la Faculté des Sciences Médicales de l'Université de Campinas, en 1975. La thèse d'Arouca a été extrêmement importante pour la construction d'une théorie sociale de la santé au Brésil, matérialisée par l'incorporation du droit à la santé dans la Constitution de 1988 et, dans les années suivantes, à travers l'institutionnalisation du système de santé publique (le Sistema Único de Saúde, ou SUS). L'originalité de la critique historique et épistémologique d'Arouca explique pourquoi sa thèse reste toujours l'une des œuvres les plus citées par les historiens brésiliens de la médecine. Pour démontrer la persévérance des thèmes canguilhemiens dans l'historiographie médicale au Brésil, nous mettrons en évidence l'œuvre de José Ricardo Ayres, professeur à la Faculté de Médecine de l'Université de São Paulo, qui dans son enquête sur l'histoire de la santé publique, en particulier dans le livre Epidemiologia e emancipação publié en 2002, annonce son "affinité méthodologique " et sa "proximité thématique» avec l'école française d'épistémologie historique. En présentant ces deux auteurs, je voudrais introduire une discussion à propos de la réception, la circulation et la transmission de quelques aspects de la pensée de Canguilhem dans le domaine le plus fertile de l'historiographie médicale brésilienne : la santé publique.

Tiago ALMEIDA

[Stagiaire de recherche à l'Université Paris I/Panthéon-Sorbonne, au sein du centre de recherches Philosophies contemporaines-Execo, sous la direction de Jean-François Braunstein. Doctorant en Histoire à l'Université de São Paulo (Brésil), sous la direction de Sara Albieri, au sein du centre de recherches Histoire intellectuelle - Intelligere, associé au Laboratoire de Théorie de l'histoire et d'Histoire de l'historiographie. Intitulé de la thèse: "L'historicisation des sciences chez Georges Canguilhem ». - Boursier de la Fundação de Amparo à Pesquisa do Estado de São Paulo - FAPESP].

\section{Between Objectivity and History: An Analysis of Ian Hacking's Styles of scientific Reasoning}

In order to defend the scientific objectivity in a historicizing way, Ian Hacking based his styles of scientific reasoning on both objectivity and the history of science simultaneously. However, on the one hand, Hacking's approach to objectivity is deeply rooted in positivism; on the other hand, his styles of reasoning are based on A.C. Crombie's studies of history of science, which belongs to the Internal-continuous tradition. As a consequence, there rise two contradictions in Hacking's styles of scientific reasoning -positivism and history, reason and society. In order to coordinate the two contradictions, Hacking constructed his important concept of selfvindication. He held that each style of reasoning is a self-autonomic whole, and that it determines its own standard of success. This concept resolved the two contradictions in his styles. However, it deals with them in an oversimplified way, and cannot offer a coherent explanation to the issue of 
objectivity and scientific evolvement. The reason is that the studies of "the history of objectivity" which the styles of scientific reasoning attach to must presuppose a concern concept of objectivity. If we distinguish "the concept of objectivity" from "the realization of objectivity", then through styles of scientific reasoning, what Hacking historicized is only the realization of objectivity. As to the concept of objectivity, Hacking's point is still ahistorical.

Wenbo LIANG

[Ph.D candidate at IHPST and Univ. Paris I: "Acupuncture anesthesia: History and epistemology”. - Supervisor: Jean Gayon (Univ. Paris I) \& Patrick Triadou (Univ. Paris 5)].

\section{PHILOSOPHIE DES MATHÉMATIQUES ET DU LANGAGE}

\section{Représentations et symbolismes en mathématiques}

Le but de cette intervention est d'examiner le rôle des représentations utilisées et du changement représentationnel en mathématiques, sur la base d'un exemple historique simple : la théorie des «configurations » du savant médiéval Nicole Oresme (env. 1320-1385), en particulier dans ses applications à la cinématique. La physique aristotélicienne ne connaît ni vitesse instantanée, ni vitesse moyenne, mais emploie une conception « holistique » (Pierre Souffrin) de la vitesse. Les «Calculateurs » d'Oxford, prédécesseurs immédiats d'Oresme (deuxième quart du $\mathrm{XIV}^{\mathrm{e}}$ siècle), introduisent une notion de vitesse instantanée et prouvent un analogue du "théorème de la vitesse moyenne" souvent attribué à Galilée. Les " configurations » d'Oresme fournissent un moyen extrêmement clair de représenter géométriquement l'évolution de la vitesse instantanée au cours d'un mouvement; elles lui permettent de présenter de manière beaucoup plus simple le travail de ses prédécesseurs. Mais il ne s'agit pas seulement d'une représentation plus claire et plus efficace de matériaux préexistants. Cette nouvelle représentation rend visible, sous la forme d'une surface, une grandeur auparavant difficile d'accès : (la " quantité totale» de vitesse) et permet de manipuler cette grandeur selon les règles de la géométrie euclidienne. Sur cette base, Oresme établit une relation radicalement nouvelle entre vitesse instantanée, temps de parcours et distance parcourue (le procédé a une parenté lointaine avec ce que nous appelons intégration). Pour finir, nous soulignons que l'emploi de ces représentations géométriques rend également accessible, pour Oresme, une autre propriété : leur « forme ». Il fonde sur celle-ci de nombreuses spéculations qui nous semblent 
aujourd'hui aberrantes, mais constituaient pour lui un emploi aussi légitime que le précédent des possibilités ouvertes par une représentation nouvelle.

David WASZEK

[Doctorant contractuel en première année. - Sujet de thèse : "Décrire les compétences inférentielles d'un agent : les représentations en mathématiques", sous la direction de Marco Panza (CNRS, IHPST). - Laboratoire de rattachement : IHPST]

\section{L’austérité du langage dans Le rapport de Brodie de Borgès}

Le rapport de Brodie est, entre autres, l'invention d'une langue : celle de la tribu des Mlch. Parmi les traits linguistiques rapportés par le missionnaire écossais David Brodie, c'est sans doute l'austérité lexicale qui pose des problèmes en philosophie du langage. Deux mots procédant par " suggestion » d'idées - plutôt que par " définitions » - suffisent à eux seuls à recouvrir un grand nombre de phénomènes : la variole, un vol d'oiseaux, la fuite qui suit une défaite, un tas de pierres, le fait de les empiler, un bois.

La viabilité de cette austérité à l'intérieur de la nouvelle réactive l'un des présupposés majeurs de la philosophie du langage, hérité de Platon et reconduit jusqu'à - et peut-être au-delà de - Wittgenstein : l'idée selon laquelle la possibilité de dire une multiplicité de choses à l'aide d'un seul mot dépendrait de la "généralité » du concept, entendue comme la saisie de quelque chose de "commun» entre des réalités différentes. La langue des "Yahoos» - nom que le missionnaire, en référence au peuple de « barbares » de l'œuvre de Swift, donne à la tribu - ne paraît «absurde » que si l'on souscrit à une position idéaliste selon laquelle leurs «idées générales» ou «concepts génériques» seraient des «abstractions dégénérées ».

On montrera qu'il existe une tension entre l'évaluation du missionnaire et les pratiques effectives de la tribu. En réalité, l'austérité de leur langue comme celle de la nôtre - est sous-tendue par une série de pratiques (intonation, gestes), par un «faire ici et maintenant» (mémoire rudimentaire, absence d'écriture) plutôt que par un « concevoir en dehors de l'espace et du temps ». La nouvelle interroge : est-il possible de coordonner une austérité lexicale en-deçà d'une conception « idéaliste » du langage ?

Une reprise narrative de deux exemples du Cahier Bleu, la parenté ethnologique entre la tribu des Yahoos et les tribus imaginées dans Le Cahier Brun, l'analogie entre cette langue et des jeux de langage des Recherches Philosophiques, et, enfin, la nature fondamentalement contextuelle des «concepts génériques » sont des éléments qui, outre qu'ils plaident pour l'hypothèse d'un Borges lecteur de Wittgenstein, suggèrent l'adhésion borgésienne - au moins dans le cadre restreint de la nouvelle, et à l'aune d'une parodie subtile du mythe de la caverne - à une position contextualiste. 
Nous tenterons de fonder philosophiquement cette lecture en restituant la situation du second narrateur: le missionnaire chrétien exprimerait la naïveté du sens commun, le «classicisme » en philosophie du langage. À vrai dire, les "concepts généraux» des Yahoos fonctionnent comme des « familles de ressemblance » wittgensteiniennes à "géométrie » différente des nôtres. Même si leurs " jeux de langage » nous sont encore inconnus, il est possible de tisser un lien entre leurs «formes de vie» et l'austérité de leur langage.

Florencia DI ROCCO

[Thèse : " Philosophie du langage et logique dans les langages fictionnels de Borges », sous la direction de Jocelyn Benoist - PhiCo (EXeCO)].

\section{MÉCANISMES PSYCHOLOGIQUES ET PHILOSOPHIE}

\section{Les processus inconscients en jeu dans la créativité et la prise de décision}

Lors de cette présentation, j'introduirai une distinction originale entre deux types de processus inconscients, et je montrerai comment ils s'articulent avec le conscient pour rendre compte de certains processus créatifs se manifestant par une intuition soudaine, ou "illumination». Une telle illumination peut se manifester pendant la veille, en interrompant la pensée en cours, ou pendant le sommeil. Elle provoque alors un réveil soudain.

Ces deux types de processus sont souvent confondus, alors qu'ils jouent des rôles fondamentalement différents et même opposés lors des processus créatifs :

- L'inconscient synchrone est le plus connu et le plus étudié. En synchronisme parfait avec la pensée et l'action, il travaille en complément des processus conscients. On le rencontre dans tous les actes de la vie consciente : il permet de parler et de comprendre le langage, il joue un rôle majeur dans les perceptions, il préside à la coordination des mouvements. Il ne joue aucun rôle créatif.

- L'inconscient autonome, quant à lui, fonctionne « en tâche de fond », sans aucun lien avec l'action ou la pensée consciente. Il peut se dérouler lorsque le sujet dort ou rêvasse, ou lorsqu'il est pleinement conscient et actif, mais en pensant à autre chose. Ainsi Poincaré a découvert les fonctions "fuchsiennes ", alors qu'il discutait d'un autre sujet. Il avait d'abord échoué dans ses recherches conscientes. Je démontrerai que ce type d'«illumination» ne peut provenir que d'un processus inconscient autonome. 
La réalité de ces processus sera démontrée et illustrée par plusieurs schémas. En les étudiant et en les comparant, je décrirai comment ils agissent pour aboutir à un résultat créatif, parfois banal, parfois génial, mais dont l'origine semble inexplicable.

Claude MAYER

[Doctorant en $2^{e}$ année en philosophie à l'IHPST (Paris I), sous la direction de Max Kistler. Le titre de ma thèse est : "Le rôle de l'inconscient dans la résolution de problèmes et la prise de décision »].

\section{La récapitulation ontophylogénétique en psychologie}

Freud applique en psychologie la théorie du biologiste néodarwinien Haeckel, d'après laquelle l'ontogenèse récapitulerait la phylogenèse. De nombreux autres psychologues et courants psychologiques utilisent également cette théorie (la psychanalyse de Freud, Jung, Erickson, le constructionnisme interactif de Piaget, le maturationisme de Gesell, l'instrumentalisme évolutionniste de Brunner, la conception pyramidale des besoins de Maslow, les stades du jugement moral de Kohlberg, la psychologie évolutionniste, la psychologie du développement, la psycholinguistique, la pédopsychiatrie, l'ethnopsychiatrie, les théories de Wallon ou Vygostsky, etc.).

Pourtant, à strictement parler, cette théorie est fausse. Elle ne vaudrait que dans certains cas précis et de manière partielle. En outre, les psychologues ne l'appliquent qu'à l'évolution de notre seule espèce, alors qu'en biologie il s'agissait de la récapitulation de l'évolution des espèces antérieures. Comment expliquer que la psychologie insiste à employer une théorie dont la biologie s'est débarrassée?

Nous posons comme première hypothèse de travail que les conditions de cette théorie sont nées en dehors du champ de la biologie, et avant elle, chez les philosophes. Dès le XVIII ${ }^{\mathrm{e}}$ siècle se mettent en place des hypothèses sur l'évolution de l'humanité par stades (Rousseau, Comte, Nietzsche ou encore Hegel).

Notre deuxième hypothèse consiste à supposer que, si les psychologues conservent cette théorie, c'est en vertu d'autres phénomènes psychologiques qui, assemblés, donnent l'impression d'une récapitulation. Il s'agit notamment de l'assimilation par l'individu des savoirs collectifs (dans l'ordre où ils ont été découverts, sachant qu'il est plus facile de les apprendre d'un autre que de les découvrir par soi-même) ; l'ordre nécessaire dans lequel se découvre la réalité (Piaget); l'emboîtement successif des opérations mentales par assimilation des concepts antérieurs (Piaget, mais aussi Husserl avec la théorie des touts et des parties).

Nous supposerons dans un troisième temps que ces mécanismes ont ensuite été globalement projetés sur le matériel philosophique, biologique puis psychologique comme apparente récapitulation. Nous reprenons alors la 
théorie freudienne de la " projection mythique », que nous avons analysée et renommée « métaformatage » (dans notre doctorat de psychologie).

Sylvain BOSSELET

[Doctorant $1^{\text {ère }}$ année. Sous la direction de Jean-François Braunstein].

\section{Causalité et philosophie de l'esprit}

La notion de cause a été et reste très débattue en philosophie de l'esprit. Je voudrais appliquer les deux notions de causalité (production et dépendance) formulées par Ned Hall (Hall, 2004) à des théories de l'esprit et de la conscience : le fonctionnalisme et le physicalisme.

La production a trois caractéristiques générales :

- la transitivité (si C cause E, et E cause F, alors C cause F) ;

- la localité (La cause est connectée à l'effet par une séquence spatiotemporelle d'événements);

- l'intrinsecness (la structure causale est déterminée par ses propriétés non-causales).

La causalité contrefactuelle a deux thèses générales :

- la dépendance contrefactuelle ;

- l'omission (l'absence d'un événement peut être cause et effet).

Hall montre que ces deux typologies de causalité sont incompatibles et que les événements sont analysables à l'aide de ces deux instruments. On verra donc en quoi les concepts de production et dépendance sont centraux dans les principales théories de la conscience et de l'esprit: le fonctionnalisme, le physicalisme et la théorie du neuro-corrélat de la conscience (NCC).

Avec l'exemple du blindsight (où il y a causalité par omission), on introduira une troisième notion de cause, selon la théorie de l'action de von Wright, qui peut être d'une grande aide pour expliquer aussi le contexte expérimental.

Marzia MicheLIZZA

[Université de Urbino (Italie) et Université Paris I. - Laboratoires : Disbef (Urbino) et PhiCo (Paris). - Directeurs : M. Mario Alai et M. Michel Bitbol. - Titre de la thèse : "Un concept phénoméniste de causalité et ses applications à la philosophie de l'esprit »].

\section{La dynamique des pensées sur soi-même}

La principale motivation de ce travail est celle de mieux connaître le processus de rétention et d'évolution des attitudes envers les pensées à la première personne. Autrement dit, j'ai l'intention de mieux comprendre ce que nous appelons la dynamique cognitive dans les cas spécifiques des 
pensées sur soi-même ou les pensées de se (en opposition aux pensées de re et de dicto). Une idée courante dans la philosophie du langage et de l'esprit est que nous pouvons être liés aux pensées en général (aussi appelées propositions, et qui sont exprimées au moyen de phrases complexes) à travers différents types d'attitudes. Par exemple nous pouvons citer l'attitude de croyance, l'attitude de doute, l'attitude d'espoir, entre autres - aussi appelées «attitudes propositionnelles». Les pensées sont ainsi comprises comme des objets de ces attitudes, et comme d'autres objets réels, nous ne les visons que diachroniquement, c'est-à-dire à travers le temps. Les cas particulièrement problématiques sont posés pour la dynamique cognitive par les pensées indexicales (exprimées au moyen d'expressions sensitives aux changements de contexte, telles que les pronoms ou les adverbes de lieu). Je défends la thèse selon laquelle les pensées à la première personne forment un cas singulier parmi les autres pensées indexicales. Par conséquent, nous devrions avoir quelque chose de particulièrement intéressant et unique à dire, pas seulement sur les pensées de se d'une manière générale, mais aussi sur le processus de penser sur soi-même. Durant cette intervention, j'exposerai d'abord l'étude de la dynamique cognitive en tant que discipline philosophique, tout en parlant également du phénomène de l'indexicalité et du rapport entre les deux. Ensuite je présenterai brièvement quelques caractéristiques propres aux attitudes de se et des pensées sur soi-même, essayant d'expliquer pourquoi nous devrions les considérer comme un cas particulier parmi d'autres types d'attitudes et de pensées. J'introduirai la métaphore des fichiers mentaux comme l'un des moyens d'expliquer la dynamique de la pensée. Enfin, dans une troisième partie, j'indiquerai de façon expérimentale quel type de voie nous pourrions emprunter pour établir une théorie essentiellement dynamiste des pensées à la première personne.

Pedro Henrique MUNIZ

[Doctorant à la Pontifícia Católica Universidade de Rio de Janeiro. - Doctorant visiteur à l'Institut d'Histoire et Philosophie des Sciences et Technique dans la cadre du projet CAPES/COFECUB Preuves, démonstrations et représentation. - Doctorant visiteur à l'Institut Jean-Nicod. - Directeur de thèse : Ludovic Soutif].

\section{LES FORMES DU MODÈLE}

\section{La réification en expérimentation animale : nécessité ou convention?}

Notre réflexion est fondée sur les discours obtenus à partir d'entretiens compréhensifs d'animaliers, de techniciens et de chercheurs au sein de l'Institut National de la Recherche Agronomique. Les personnes entretenues sont en relation directe avec les animaux et dans une posture 
d'expérimentation animale. Cette posture est spéciste car elle repose sur « une attitude de parti pris en faveur des intérêts des membres de sa propre espèce et à l'encontre des intérêts des membres des autres espèces " (Singer, 1975). Les intérêts de l'espèce humaine sont privilégiés, qu'il s'agisse d'expérimentations qui favoriseront la productivité des animaux d'élevage ou bien d'autres qui utilisent des animaux " modèles " pour des maladies qui concernent l'homme (Francione, 2000). La mise à distance de l'animal utilisé pour l'expérimentation est inscrite dans la majorité des formations professionnelles qui placent le professionnalisme dans la technique et le refus de l'affect (Jeantet, 2003) ; cette attitude est très présente en médecine humaine (Fainzang S., 2006). Cette mise à distance est également fondée sur le fait que l'animalier reçoit une prescription du chercheur, qui lui-même ne verra pas toujours l'animal mais ses traductions expérimentales (prises de sang, de fèces, etc.). La législation relative à l'expérimentation animale (Arrêté du 9 décembre 2014), même si elle a produit des améliorations sensibles des animaux, a aussi des effets de mise à distance : suivre les recommandations suffit. La réification de l'animal d'expérience est de type intersubjective-dans le rapport aux autres (Honneth, 2007) et se base sur l'oubli préalable de la reconnaissance de l'autre. La réification de l'animal nous semble plus une convention commode (Porcher, 2011) pour les expérimentateurs plutôt qu'une nécessité. Une tension entre l'animal outil (relation « dictée » pour l'animalier) et l'animal proche (relation personnelle avec celui qui prodigue les soins, situation de care (Fisher et Tronto, 1990)) est réelle et avait été également observée par Lhoste et de Monteira (2011). Cette tension éclairera l'importance respective de la convention et de la nécessité.

Jacques CABARET

[INRA, Infectiologie et santé publique, Bat. 213, 37380 Nouzilly-F. - Directeur : Jean Gayon (IHPST, Paris I/Panthéon Sorbonne). - Sujet de thèse : "Le concept de maladie animale et ses conséquences thérapeutiques ». - Ce résumé est le résultat d'un travail conduit avec Ludivine Fortin (qui était en M2 Ethires, philosophie et éthique, Paris I/PanthéonSorbonne)].

\section{Analogue naturel et transition énergétique}

La notion d'analogue naturel renvoie à un contexte particulier, celui de la recherche dans les sciences de la nature. Cette notion est utilisée dans de nombreux projets de transition énergétique. Nous nous intéresserons dans cette thèse à trois d'entre eux : un projet de géo-ingénierie qui consiste à injecter des particules de soufre dans la stratosphère pour «refroidir» le climat; un projet de stockage de $\mathrm{CO}_{2}$ dans des couches géologiques profondes pour diminuer la concentration de ce gaz dans l'atmosphère ; enfin un projet de production d'hydrogène pur à faible coût énergétique. Dans ces projets, des phénomènes naturels (respectivement éruptions 
volcaniques, accumulations de $\mathrm{CO}_{2}$ dans certaines couches géologiques et serpentinisation) sont appréhendés comme des analogues naturels du projet technique que l'on cherche à mettre en place.

La thèse engagée vise à mener une analyse réflexive sur l'usage pratique des analogues en mettant à jour tout ce qui est impliqué et présupposé dans ce travail de rapprochement entre un phénomène naturel observé et un projet technique. Les prémisses de nos recherches montrent que les analogues naturels sont des pistes possibles de résolution des problèmes énergétiques, des supports d'informations pour appréhender les incertitudes et risques associés à ces projets et parfois même des arguments pour favoriser leur réalisation. Le recours aux analogues naturels serait, dans ce dernier cas, une façon de légitimer un projet technique aux yeux du public (si la nature le fait, alors sans doute peut-on le faire !).

Le dialogue avec des chercheurs impliqués dans les trois projets évoqués nous permettra de préciser ces usages pratiques. Pour exemple, s'agit-il d'imiter, c'est-à-dire de dégager le principe général à l'œuvre dans le phénomène naturel pour ensuite tenter de le transposer en respectant les normes propres au design technique? Jusqu'à quel point pouvons-nous confondre l'analogie envisagée avec l'imitation en tant que transfert de technologie comme dans le cas du biomimétisme? Ces trois projets conduisent aussi à s'interroger sur le sens des mots " nature » et «naturel» ainsi qu'à revisiter la question traditionnelle des rapports entre la nature et l'artificiel. La relation d'analogie établie modifie-t-elle la perception du processus naturel?

Pour finir, notre étude cherchera à définir le type de relation qui s'est construit entre chercheurs en sciences de la nature et chercheurs en sciences humaines sur ces projets de société.

Dorine MONTOUT

[Directeur de thèse: Bernadette Bensaude-Vincent. - Laboratoire de rattachement: CETCOPRA. - Titre de la thèse : "Entre nature et technique : enquête épistémologique sur le recours aux analogues naturels face à la transition énergétique »].

\section{La construction ingénieuse du modèle chez Baltasar Gracián}

Gracián passe souvent pour l'auteur de traités de cour qui chercheraient à proposer, de manière tout à fait classique, des modèles de réussite sociale. Il dessine en effet, dans des textes aux titres révélateurs (Le Héros, Le Discret, Le Politique) des types théoriques qu'il s'agit non seulement d'admirer mais aussi d'imiter. De la même manière, dans son traité de rhétorique, La Pointe ou l'art du génie, il propose un recueil ordonné d'exemples de figures de styles empruntés aux grands poètes qui constituent autant de modèles de traits d'esprit réussis. Mais la pensée au modèle ne se réduit pas chez Gracián à une simple considération rétrospective d'objets 
préexistants. La pédagogie du modèle ne consiste pas seulement à se former sur un modèle, mais bien à se former comme un modèle. Le modèle n'est pas d'abord la cause mais plutôt l'effet de l'admiration. Devenir un " héros » sur la scène du monde ne suppose pas tant d'être en conformité avec le patron du type vertueux, que de parvenir à apparaître comme héroïque aux regards des autres. De la même manière, une figure de style ne devient un modèle poétique que dans la mesure où elle «frappe » l'esprit du lecteur sans nécessairement correspondre à un horizon d'attente déterminé. Dans les deux cas, le modèle est toujours une construction de l'esprit (ingenio). Or c'est précisément la manière dont se construisent les modèles que nous souhaitons interroger. Alors même que le champ social est un univers agonistique où les succès sont toujours précaires et où les renommées se font et se défont, comment procéder à cette construction de soi comme modèle inaltérable?

Nous nous demanderons si la réponse à cette question n'implique pas ultimement, chez Gracián, de penser le monde humain sur le modèle général $\mathrm{du}$ livre, et de penser ainsi les figures de style comme autant de figures de l'existence. C'est alors dans sa fonction analogique et allusive que nous pourrons trouver l'unité théorique du concept de modèle chez Gracián.

Thibault BARRIER

[Sujet de thèse: "Le temps de l'admiration: surprise et nouveauté aux XVI et XVII siècles », sous la direction de Chantal Jaquet. - Laboratoire : CHSPM].

\section{Le modèle et la norme dans le Discours philosophique de la modernité d'Habermas}

On se proposera ici d'interroger la notion de «modèle » à partir de sa confrontation à celle de "norme ». Quel type de normativité est à l'œuvre dans l'exemplarité du modèle? Cette question révèle son importance lorsqu'on cherche à thématiser le rapport des modernes à l'histoire. Depuis Hegel en effet, la modernité a été thématisée comme une rupture assumée avec l'histoire exemplaire, c'est-à-dire avec une représentation de l'histoire comme un réservoir de modèles anciens qu'il s'agirait de reproduire ou d'imiter. C'est autour d'une telle compréhension que se construit le Discours philosophique de la modernité d'Habermas, qui pense la modernité comme le passage d'une normativité exemplaire et hétéronome à une normativité auto-fondée, immanente car produite par la réflexion collective, et donc autonome. La modernité a ceci de spécifique qu'elle développe une conscience historique du temps qui lui permet de trouver en elle-même «ses propres garanties». La critique de l'histoire exemplaire qu'on trouve chez Habermas s'avère ainsi mobilisée à partir de ce qui en elle converge vers une critique de toute normativité hétéronome, imposée de l'extérieur aux acteurs, et qui ne serait pas l'objet d'une réappropriation par la réflexion 
collective. On reconstruira cette opposition implicite du modèle et de la norme afin de se demander si Habermas nous donne par là accès à une critique des modèles. Il s'agira d'interroger le statut de cette critique dans l'économie de son propos.

Clotilde NOUËT

[Doctorante en cotutelle sous la direction de: Jean-François Kervégan (Paris I) et Rahel Jaeggi (Humboldt-Universität zu Berlin). - Laboratoire : PhiCo-NoSoPhi. - Sujet de thèse : "Critique du capitalisme et normativité, la théorie sociale de Jürgen Habermas »].

\section{Instituer et destituer des modèles. La précarité des alliances chez Nietzsche}

On sait que l'itinéraire philosophique de Nietzsche est intimement attaché aux manières qu'il a eu de se confronter à des physiologies. Qu'elles soient celles de ses contemporains, comme Wagner, Jakob Burckhardt, Hippolyte Taine, Emerson, ou celles des grands (et des moins grands) du passé : Platon, Machiavel, Schopenhauer, etc., c'est toujours, avec Nietzsche, à une confrontation personnelle que l'on assiste, en ceci que les alliances et les rivalités se constituent moins sur un plan conceptuel chez lui que sur un terreau psycho-physiologique. Les noms de l'histoire sont d'abord des types, où s'individualisent des matrices pulsionnelles propres à certaines tendances de l'histoire de la culture. Comment, à partir de cette typologie - elle-même pensée dans son rapport à certains modèles épistémologiques: la morphologie holiste de Goethe, la morphologie de l'histoire universelle de Burckhardt, la typologie de Taine ou encore certaines tendances de la Volkspsychologie -, Nietzsche statue-t-il sur la vocation axiologique des modèles et contre-modèles à constituer des motifs d'auto-dépassement ou des repoussoirs pour la volonté de puissance ? Et surtout, sous quelles modalités ces opérations de détournement (du contremodèle) ou d'appropriation et dépassement (du modèle) s'effectuent-elles ? L'examen du trajet intellectuel de Nietzsche permettra de mettre au jour les stratégies par lesquelles un discours philosophique s'auto-historicise d'abord à travers des modèles pour penser sa vocation transhistorique posthume : en quel sens Nietzsche peut-il lui-même devenir un modèle pour nous sans procéder pour autant à sa propre canonisation?

Arnaud SOROSINA

[Directeur : Bertrand Binoche (CHSPM). - Titre de la thèse : "Les historicités de Nietzsche. Une perspective génético-généalogique »]. 


\section{PhILOSOPHIE SOCIALE ET POLITIQUe}

\section{Démocratiser le travail. Ce que nous apprennent les expériences autogestionnaires}

Depuis plusieurs siècles, le mouvement coopératif ambitionne d'inscrire l'activité politique au cœur de notre quotidien notamment en faisant du travail une organisation démocratique en elle-même. Les coopératives de production sont ainsi des entreprises détenues par leurs travailleurs, qui décident collectivement des fins et des moyens de leur entreprise selon le principe «une personne, une voix». Dans les années 1970, le concept d'autogestion s'appuie sur des idées spécifiques par rapport au mouvement coopératif, principalement sur l'idée de démocratie directe ainsi que sur la valorisation d'une gestion décentralisée du pouvoir politique. Cependant, nous soutenons que les expériences autogestionnaires auxquelles renvoient ce concept d'autogestion ne diffèrent que très peu sur le plan du fonctionnement politique des coopératives de production: on y observe presque systématiquement une dynamique d' "atrophie-oligarchie » à l'œuvre. Nous montrons dans un second temps qu'il est simpliste de proposer une explication de cette dynamique, qui a souvent valeur de justification, en termes de stratégie de la part des salariés qui prennent le moins part à la vie politique de l'entreprise. Il faut au contraire non seulement penser les phénomènes de monopolisation du pouvoir de la part d'une minorité mais aussi la problématique de la reproduction des inégalités sociales plus générales comme étant un facteur-clé de la dégénérescence politique de ces organisations. Face à ces problématiques, nous présentons enfin deux pistes normatives : la revalorisation de la rotation des postes et la redéfinition du rôle des syndicats.

Camille TERNIER

[Assistante-doctorante en philosophie à l'université catholique de Lille. - Mes deux directeurs de recherche sont Malik Bozzo-Rey (Université catholique de Lille) et Emmanuel Picavet (Paris I). - Sujet de thèse: "Valeurs et processus démocratiques dans les organisations de l'Économie Sociale et Solidaire : le cas des coopératives de production »].

\section{La Théologie politique et l'histoire des concepts : le cas de Carl Schmitt, à travers Hans Blumenberg et Reinhart Koselleck}

Ma présentation discute certaines idées de théologie politique de Carl Schmitt. Ce que je voudrais faire ici c'est, d'abord, d'examiner les idées de sécularisation chez Schmitt et les critiques qu'en fait Hans Blumenberg; puis, de démontrer la pertinence de ce débat pour le développement de la «sociologie des concepts politiques» de Schmitt à travers «l'histoire conceptuelle» de son élève, Reinhart Koselleck. 
Nous conclurons sur une distinction entre les facettes monothéiste et polythéiste de la théologie politique et nous examinerons les conséquences de l'approche méthodologique implicite de Schmitt chez Koselleck en particulier. Nous projetons d'analyser les angles sous lesquels il sera possible de formuler différentes questions contradictoires sur la théorie politique de Carl Schmitt et d'exposer certaines ambiguïtés essentielles concernant le politique. À partir des ambiguïtés trouvées, nous examinerons les dynamiques historiques à l'œuvre dans la pensée théologico-politique de Schmitt. Ceci nous permettra de mettre en lumière les processus implicites de clarification du politique. Cette analyse suggère que cela me mènera à voir la théologie politique comme histoire des concepts d'amitié (et d'inimitié) et d'émancipation (ainsi que du salut et du miracle).

Masoud SINAEIAN

[Titre de la thèse: "Théologie politique, histoire et méthode chez Carl Schmitt ». Directeur de recherche: Jean-françois Kervégan. - Laboratoire: Centre de philosophie contemporaine de la Sorbonne (NoSoPhi)].

\section{Que critique Adam Ferguson dans la division du travail ?}

Adam Ferguson (1723-1816) est une figure des Lumières écossaises, sinon oubliée, du moins largement méconnue de nos jours. Pourtant, c'est bien dans son œuvre et non dans celle, plus célèbre de Smith, que Marx voit la première réflexion sur les conséquences fâcheuses de la division du travail, faisant même, par erreur, de Ferguson, le maître de Smith ${ }^{1}$. Le terme « division du travail » (division of labour) n'est cependant pas présent dans An Essay on the History of Civil Society, auquel Marx fait référence. Cela ne doit pas nous tromper: si le mot n'y est pas, la chose elle-même n'en reste pas moins thématisée. Jean-Pierre Séris lui a d'ailleurs consacré un petit ouvrage $^{2}$, au sein duquel il remarque la présence du verbe subdivide (subdiviser), terme clairement technique, qui fait référence à la division du travail. En outre, ce thème est véritablement récurrent dans les écrits de Ferguson. Il en est question dans An Essay on the History of Civil Society (1767), mais aussi dans les Institutes of Moral Philosophy (1773), dans les Principles of Moral and Political Science (1792) et, après 1806, dans un manuscrit non publié ${ }^{3}$.

Or, la réflexion de Ferguson au sujet des effets de la division du travail, du commerce et de l'industrie, ne cesse d'être ambiguë. Ceci a conduit les lecteurs de Ferguson à proposer des interprétations très différentes de son œuvre. Si Marx voit en lui un proto-marxiste, F. A. von Hayek le reconnaît

1. Misère de la philosophie, Ch. II, $\S 2$.

2. Jean-Pierre Séris, Qu'est-ce que la division du travail ? Ferguson, Paris, Vrin, 1994.

3. The Manuscripts of Adam Ferguson, London, Pickering and Chatto, 2006. Le manuscrit: "Of the Separation of Departments Professions and Tasks Resulting from the Progress of Arts in Society” traite de ce sujet. L'éditeur V. Merolle indique que le papier utilisé date de 1806. 
bien plutôt comme un libéral classique, un penseur de l'ordre spontané, dont il se fait lui-même le chantre. Cette ambiguité, mise en lumière par des lectures aussi différentes, est bel et bien présente dans l'œuvre même d'Adam Ferguson. Les louanges sincères faites à la division du travail, condition sine qua non du passage de l'état « rude» des premiers âges à un état policé, à la civilisation des sociétés commerçantes, s'accompagne d'une critique non moins sévère, lorsque celle-ci conduit à l'atrophie des facultés et à la perte des vertus civiques. La division du travail, effet non intentionnel des actions humaines, est ainsi productrice de civilisation et d'ordre et, dans le même temps, productrice de désordre et de corruption. Ferguson associe d'ailleurs la superstition et l'industrie, comme étant toutes deux filles de l'ignorance ${ }^{4}$.

Le but de la présentation du 4 juin 2015 était de montrer que Ferguson n'est pas le critique idéologique de la modernité que d'aucuns ont cru identifier dans ses écrits. Sa réflexion est bien plutôt construite à partir d'une attention particulière à la psychologie morale de l'agent, que l'on peut lire en filigrane dans son œuvre. J'ai argumenté que ce qui est dénoncé dans la division du travail repose sur le caractère corrupteur qu'elle peut avoir sur l'imagination, privant l'agent de sa capacité à inventer et à aimer, si ne sont mises en place des activités pouvant y remédier. Je crois que Ferguson voyait dans le jeu, dont le paradigme est l'exercice militaire au sein de la milice, une solution aux problèmes engendrés par la division du travail. Ferguson associe, en effet, la liberté du jeu à la formation d'un esprit libre et à l'exercice par l'homme de toutes ses capacités. Ce serait donc principalement le jeu au sens large qui créerait les conditions de la culture de l'imagination, offrant une certaine qualité de l'agir, garante de la liberté civile.

Sonia BOUSSANGE-ANDREI

[Doctorante en deuxième année à l'université Paris I/Panthéon-Sorbonne (PhiCo/NoSoPhi). - Université de Neuchâtel sous la direction de Laurent Jaffro et de Daniel Schulthess. - Sujet de la thèse : "Les rapports entre morale et société dans la pensée d'Adam Ferguson »].

4. An Essay on the History of Civil Society, Part IV, Sect. I. 


\section{AUTOUR DE LA GUERRE : REGARDS CROISÉS}

\section{Les causes des guerres civiles et étrangères chez Platon}

\section{«[...] l'âme est la source d'où découlent pour le corps et pour l'homme entier tous les biens et tous les maux $[\ldots]^{5} »$.}

\section{L'appel à la psychologie.}

Pour comprendre l'homme chez Platon, il faut partir de l'âme, car l'âme est le principe de la vie. La psychologie platonicienne cherche à comprendre l'âme, sa nature, ses errements. L'homme est union de l'âme et du corps. Par le fait que l'homme possède un corps et par le fait qu'il a des besoins, il devient un être de désirs. Les désirs doivent être tempérés, malheureusement, tel n'est pas toujours le cas.

\section{Les racines des guerres.}

Pour Platon, c'est toujours une mauvaise raison qui est à l'origine des guerres civiles et étrangères (la guerre offensive s'entend). Les guerres existent à partir du moment où les âmes humaines sont en proie aux richesses de ce bas-monde.

Guerre civile.

C'est la pire des guerres puisqu'elle oppose les citoyens d'un même pays. Elle vient au jour lorsque l'unité de la cité est menacée par les dissensions et les calomnies.

\section{Guerre étrangère.}

Platon est contre la guerre offensive. Il prône la coexistence pacifique. La guerre étrangère vient à jour lorsque la coexistence pacifique est menacée par la pléonexia (désir d'avoir trop au mépris de toute raison). La pléonexia se traduit par l'empiétement du domaine propre de l'autre.

Mamadou SOUMARÉ

[Professeur-certifié de philosophie, Sénégal. - Doctorant sous la direction du Professeur Luc Brisson. - Projet de recherche doctoral : "Le problème de la guerre dans les dialogues de Platon ». - Laboratoire : GRAMATA].

5. Platon, Charmide, 156e-157a, in Euvres complètes, t. 2, trad. par Alfred Croiset, Paris, Les Belles Lettres, (Coll. Budé), 1965. 


\section{La guerre pour les sujets d'un autre chez Grotius. Du droit de punir à la permission d'intervenir}

(Commentaire du $\S$ VIII, ch. 25, livre II du Droit de la guerre et de la paix de Grotius)

Grotius dans le Droit de la guerre et de la paix de 1625 énonce les cas dans lesquels l'entreprise de la guerre est compatible avec le droit. À l'intérieur de ce projet l'auteur signale le cas de la guerre entreprise par un prince pour mettre fin à l'oppression qu'un souverain étranger fait subir à son propre peuple. Bien qu'il semble ne lui accorder qu'une place mineure, elle n'est abordée qu'à la toute fin du livre II -, il demeure qu'elle occupe dans l'économie de l'ouvrage un rôle fondamental. Nous essayerons de montrer que l'analyse de cette guerre est essentielle pour saisir la conception de la souveraineté propre à l'auteur. En effet, la guerre pour les sujets d'un autre le conduit à la fois à renforcer l'absoluité des pouvoirs qu'il confie au prince, mais aussi à restreindre la liberté qu'il lui accorde à l'intérieur de ses frontières. Il s'agira d'étudier la manière dont l'auteur tente de faire de la guerre de libération un cas de guerre juste par la recherche d'un fondement moral et juridique.

C'est dans le mouvement même par lequel Grotius renforce la souveraineté du prince qu'il réussit à construire une limite en autorisant le renversement par la guerre pour les sujets d'un autre. L'auteur fait de la mobilisation injuste du droit de punir, pourtant premier droit du souverain car à l'origine de l'institution de la société politique, l'assise morale permettant de constituer cette forme de guerre. Le fondement juridique, lui, est trouvé dans le droit de la société humaine. Grotius va faire du droit du regroupement pré-politique, la source de légitimité de la guerre de libération. Ce n'est pas le droit naturel de l'individu qu'il oppose au pouvoir du tyran, mais celui de la communauté unie avant la fondation du politique, appui juridique sur lequel la guerre pour les sujets d'un autre pourra s'établir.

Lyess BOUDERBALA

[Titre de la thèse: "Les apories de la guerre juste à l'âge classique: intervention, souveraineté, autodétermination ", sous la direction de Bertrand Binoche, au CHSPM].

\section{Le rôle des intellectuels dans les transformations politiques et sociales de la société kurde après l'apparition de l'opposition en 2009 au Kurdistan irakien}

Le rôle des intellectuels dans les transformations politiques et sociales dans la société kurde nous paraît, pour diverses raisons, très important. Les intellectuels kurdes, en raison de l'histoire et de la nature de la composition de la société kurde, ont leur propre particularité. Il nous a donc paru important de les distinguer des autres intellectuels. Il est clair que les 
intellectuels ont toujours participé, d'une façon ou d'une autre, à la libération du Kurdistan.

Une partie de notre travail devait être consacré au climat culturel qui existe dans la société kurde. Quelles sont les organisations culturelles et leur rôle dans une société musulmane ? Comment ces institutions fonctionnentelles ? Quelle est la place des organes des médias et de l'éducation de l'enseignement supérieur au Kurdistan et ont-ils une influence sur les intellectuels? Les syndicats et les instances juridiques jouent-ils un rôle au Kurdistan?

Nous avons choisi une période bien précise allant de 1991 à 2011 qui nous permet de bien distinguer les différentes situations après le soulèvement de 1991. Ensuite, nous avons consulté de nombreux journaux, revues spécialisés ou non dans lesquels parfois des informations importantes figuraient, ce qui fut également le cas pour un certain nombre de sites internet. Nous avons pu aussi visionner quelques programmes télévisés (des rencontres et débats politiques avec les intellectuels).

Afin de mieux réaliser ce travail, nous avons fait une enquête de terrain au Kurdistan, mais uniquement dans les deux villes de Suleimany et Erbil. Pour des raisons de sécurité, nous n'avons pas pu aller à Kirkuk pour nous y entretenir avec des intellectuels. Ce fut le manque de temps qui ne nous a malheureusement pas permis de rencontrer les intellectuels de Dehuk. C'est pourquoi notre recherche se limite uniquement aux deux principales villes. Nous avons pu rencontrer des intellectuels de toutes les différentes catégories que nous avons cités plus haut.

Shawnm Yahya KIDIR

[Titre de la thèse: "Le rôle des intellectuels dans les transformations politiques et sociales après l'apparition de l'opposition en 2009 au Kurdistan irakien », sous la direction du Professeur Jean Salem].

\section{Une militarisation des savoirs : de l'usage stratégique des sciences humaines durant la guerre d'Algérie}

J'aimerais proposer ici une illustration historique de la problématique foucaldienne du rapport entre savoir et pouvoir à partir de l'exemple d'un usage tout à fait singulier des sciences humaines dans les pratiques de l'armée française durant la guerre d'Algérie. Il s'agira ici d'explorer la généalogie d'une militarisation de la production de savoirs ainsi que de pouvoir interroger les influences d'une dimension stratégique dans ce processus de production.

Cet ancrage historique se fondera sur les pratiques des Services Administratifs Spécialisés (S.A.S.), mis en place en Algérie en 1955, et de leur version métropolitaine, le Service d'Assistance Technique aux Français Musulmans d'Algérie (S.A.T.-F.M.A.), mise en place à la Préfecture de 
police de Paris en 1958 par Maurice Papon. Ces services, chargés d'organiser un réseau de surveillance militaire de la population algérienne à partir d'une activité socio-administrative d'assistance, ont la particularité d'avoir mobilisé dès leur création des savoirs divers issus à la fois d'une anthropologie coloniale qui trouve ses origines dès le début du $\mathrm{XX}^{\mathrm{e}}$ siècle, d'une psychologie militaire qui s'illustre dans les doctrines de la guerre psychologiques et de l'action psychologique, ou encore d'une sociologie urbaine, du Maghreb notamment.

Cette transdisciplinarité tactique nous conduit tout d'abord à poser la question des conditions d'élaboration de certains savoirs destinés à l'application de pratiques de gouvernementalité tant dans une visée préventive de surveillance que dans une visée pro-active d'anticipation. Cette mobilisation des savoirs permet d'illustrer en quoi des sciences telles que la sociologie ou encore l'anthropologie sont susceptibles de devenir des outils propres de pouvoir et de gouvernement, et permettent d'introduire une légitimité scientifique aux pratiques policières d'identification, de catégorisation et de classification; pratiques essentielles à cette économie du maintien de l'ordre.

Nous proposons ainsi de nous confronter à ce moment historique pour voir en quoi de telles pratiques policières nous renseignent sur un agencement singulier du rapport entre production de savoir et pratiques de pouvoir, mais aussi sur la manière dont, en temps de guerre, les pratiques administratives peuvent constituer un champ d'expérimentation. Cette valeur expérimentale semble émerger d'un déplacement du champ des possibles guidé par une rhétorique de l'urgence permanente à partir de laquelle l'exception devient une référence normative. Dans de ce contexte, il s'agira d'interroger les conséquences de ce déplacement, c'est-à-dire les pratiques issues de cet état d'exception et la manière dont elles ont pu s'inscrire alors dans des pratiques administratives de contrôle des migrations postcoloniales.

Théophile LAVAULT

[Membre de PhiCo, doctorat sous la direction de Judith Revel. - Sujet de thèse: "Police administrative et dispositifs de contrôle de l'immigration. Une monographie du Service d'Assistance Technique de la Préfecture de police de Paris (1958-1984) »]. 


\section{PERSistanCE ET INDIVIDUALITÉ DES ÊTRES VIVANTS}

\section{La stabilité des vivants : comparaison des régimes d'auto- maintien des organismes et des écosystèmes}

La persistance des vivants est problématique en ce que les êtres vivants sont hors de l'état d'équilibre thermodynamique. Dit simplement, un être vivant est un système qui se maintient activement dans son état de vie contre une tendance naturelle à la corruption. La théorie des systèmes dissipatifs de Prigogine explique que cela est possible en raison de ce que tout vivant est traversé par un flux d'énergie et de matière (Nicolis et Prigogine, 1977). Pour reprendre Bichat, un organisme est un «ensemble de fonctions qui résistent à la mort », et nous ajoutons, qui dépendent mutuellement les unes des autres. Nous nous plaçons ainsi dans une tradition remontant à Kant (1790) qui explique la possibilité de cet état singulier de la matière qu'est la vie au moyen du concept d'organisation, défini en termes d'autoproduction (Varela et al, 1974) et plus récemment de clôture de contraintes. (Rosen, 1991 ; Moreno et Mossio, 2015). Au-delà de la possibilité même de son existence, la vie interroge par sa persistance face à l'adversité. Les organismes sont robustes en ce sens qu'ils sont capables de se maintenir face à des perturbations. Cette robustesse est le produit de régulations, fonctions de second ordre permettant de basculer entre différents régimes de fonctionnement selon les besoins.

L'objet de notre communication fut de déterminer si la stabilité des écosystèmes pouvait recevoir une explication analogue à celle de la stabilité des organismes. Les écosystèmes sont également des systèmes dissipatifs composés de parties auxquels les écologues attribuent des rôles fonctionnels. Ces parties sont notamment les populations biologiques qui, au niveau de description le plus général, se voit attribuer les rôles de producteurs primaires (végétaux), de consommateurs primaires (herbivores), de consommateurs secondaires (prédateurs), et de décomposeurs. Nous avons défendu qu'il était plausible que ces parties entretiennent des relations de dépendance mutuelle telles que les écosystèmes entrent dans la catégorie des êtres organisés. À l'instar de celle des organismes, leur organisation - si elle existe - est robuste. Cependant, les écologues expliquent cette robustesse au moyen d'une hypothèse de redondance fonctionnelle plutôt que d'une hypothèse de régulation. Les espèces remplissant un même rôle fonctionnel au sein d'un écosystème agiraient comme des " assurances biologiques ». Pour un paramètre considéré de l'environnement, chacune aurait son propre optimum. Si ce paramètre environnemental est modifié, l'abondance relative des différentes espèces fonctionnellement redondantes serait modifiée de telle sorte que la fonction de l'écosystème serait toujours assurée 
avec le même niveau de performance. L'écosystème modifierait ainsi quantitativement ses parties pour perdurer en tant que totalité. En passant à la limite, cette capacité devient celle de modifier qualitativement ses parties afin de perdurer, soit la capacité de se réguler. La redondance fonctionnelle apparaît ainsi comme une condition préalable à l'apparition de la régulation au cours de l'évolution de la vie. En comparant organismes et écosystèmes, nous pouvons donc dégager des degrés successifs d'organisation biologique : dépendance mutuelle, puis redondance fonctionnelle, et enfin régulation.

\section{Références}

Kant Immanuel, Critique de la faculté de juger, Paris, France, Aubier, 1995.

Moreno Alvaro \& Mossio Matteo, The Autonomy of living systems: a philosophical enquiry into biological organization, Springer, 2015.

Nicolis G \&t Prigogine I, Self-organization in nonequilibrium systems: from dissipative.structures to order through fluctuations, Wiley, 1977.

Rosen Robert, Life itself: a comprehensive inquiry into the nature, origin, and fabrication of life, Columbia University Press, 1991.

Varela Francisco, Maturana Humberto \&t Uribe Ricardo, « Autopoiesis: The Organisation of Living Systems, its characterization and a model », BioSystems, 1974, vol. 5, p. 187-196.

Victor LEFÈVRE

[Thèse: "L'écosystème, un quasi-organisme? Valeur scientifique et conséquences philosophiques de l'organicisme en écologie ». Sous la direction du professeur Jean Gayon, Université Paris I Panthéon-Sorbonne / Institut d'Histoire et de Philosophie des Sciences et des Techniques (IHPST)].

\section{La robustesse des organismes comme critère d'individualité}

La « robustesse » (robustness) est la capacité d'un système à persister suite à des perturbations externes et internes et de maintenir ses fonctions. Il s'agit d'un concept très important en physique, ingénierie et chimie, qui devient également important en biologie aujourd'hui.

Le concept de «robustesse » semble être le concept scientifique qui rend le mieux compte du concept philosophique d'identité diachronique, c'est-à-dire l'idée qu'un individu est quelque chose qui a une identité qui persiste au cours du temps tout en changeant. Établir ce qui fait l'identité diachronique est encore plus important dans le cas de l'individu biologique, qui change beaucoup durant sa vie.

Dans mon exposé j'essayerai, en particulier, d'appliquer la notion de « robustesse» à l'organisme : dans ce cas, il s'agit de la capacité de l'organisme à maintenir ses caractéristiques phénotypiques fondamentales à travers les variations internes (génétiques) ou externes (environnementales). En effet, un organisme peut subir des changements génétiques aléatoires 
pendant son développement; mais il doit aussi survivre aux changements de l'environnement dans lequel il vit (par exemple, changements de température ou de salinité). L'organisme est donc robuste s'il tolère tous ces changements, parfois en résistant et parfois en s'adaptant.

À partir de l'analyse de la «robustesse» des organismes, on se demandera si elle peut devenir l'un des critères d'individualité : c'est-à-dire, si un système biologique pour être un individu doit être robuste. Et aussi, on verra si le fait que cette notion s'applique à différentes entités biologiques (de l'organisme à l'écosystème) implique qu'elles sont toutes des individus biologiques.

Dans ce sens, mon exposé veut donc établir le lien entre le concept de « robustesse » tel qu'il est conçu dans les sciences et la définition d'individu biologique comme quelque chose qui dure dans le temps.

Anna Maria DIELI

[Thèse en philosophie des sciences en cotutelle: Università degli Studi di Roma "Tor Vergata”, et Université Paris I Panthéon-Sorbonne. - Directeurs de thèse : Giovanni Iorio Giannoli ("Tor Vergata") et Philippe Huneman (IHPST). - Sujet de recherche: "La question de l'individualité en philosophie de la biologie »].

\section{Complexité et phase de constitution des corps chez Spinoza}

«Pour que les scientifiques en général parviennent à comprendre peu à peu le monde, il faut non seulement que ce dernier soit "compréhensible", mais aussi que les hommes, identifiés par cette aptitude et ce mode humains de compréhension, soient eux-mêmes explicables dans les mêmes termes qui servent à expliquer les processus du monde, en tant précisément qu'ils font partie de celui-ci $»^{6}$. Prise en rigueur, cette phrase aurait pu être dite par Spinoza lui-même, ou tout du moins par un de ses sectateurs ayant lu l'Appendice de la Partie I de l'Éthique. Faustino Cordón, sans préciser le nom de celui qu'il appelle seulement " un moniste rigoureux », ne dit pas si l'analyse de cette " capacité humaine d'accès au connaître » est héritée $\mathrm{du}$ penseur $\mathrm{du} \mathrm{XVII}^{\mathrm{e}}$ siècle. Cela n'est finalement pas important. Il est néanmoins primordial de comprendre comment en mobilisant un programme «de monisme scientifique intégral» sur la base de modes constitués et explicables en termes d'aptitudes organiques, ce chercheur valide et active un cadre théorique doublement importé (d'une époque et d'une discipline différentes). Si la filiation spinozienne n'est pas centrale, ce haut degré d'usage de concepts et de perspective spinoziennes doit nous interroger sur l'heuristique proprement scientifique de certaines notions d'origine ontologique. En retour, l'addition de termes tirés d'un contexte biologique récents peut peut-être élargir et préciser le cadre théorique en question.

6. Faustino Cordón, «Fondements biologiques d'une théorie de la conscience », in Patrick Tort (dir.), Pour Darwin, Paris, PUF, 1997. 
Nous pensons par exemple que les concepts de pulsations discrètes ou de phases de composition éclairent rétrospectivement mais directement la conception spinozienne des degrés de composition des corps. À l'appui des développements issus de l'analyse de Cordón, nous tenterons donc de comprendre comment la notion de composition corporelle n'a rien perdu de son tranchant en se déplaçant de son versant ontologique vers une utilisation purement biologique.

Vincent LEGEAY

[Doctorant au CHSPM (EA 1451) sous la direction de Chantal Jaquet. Il travaille sur la portée historique du concept d'aptitude chez Spinoza et son actualité dans la biologie moderne et contemporaine].

\section{La distinction entre action parfaite et mouvement inachevé en Métaphysiques $\Theta-6$}

Dans cette intervention, je voudrais premièrement présenter une distinction élaborée par Aristote dans un passage du livre $\Theta$ des Métaphysiques, au chapitre 6, entre l'acte (energeia) et le mouvement (kinesis): certains mouvements contiennent en eux leur fin, leur accomplissement, et d'autres tendent vers une fin extérieure, ils sont inaccomplis. Les mouvements qui contiennent leur fin sont appelés actes, et les exemples donnés sont des opérations vitales telles que connaître, voir, vivre, être heureux. Les mouvements inaccomplis sont considérés comme mouvements au sens strict, et continuent pour cela d'être appelés mouvements. Les exemples donnés sont aussi des opérations vitales: apprendre, guérir, manger, maigrir, marcher, construire. Je regarderai aussi les distinctions qui semblent similaires en De l'âme II-5 et Physique III. Je crois que cette distinction peut fournir une manière de comprendre comment un être vivant doit changer pour rester le même, car les mouvements inaccomplis ont comme résultat un changement dans leur sujet mais ils sont en vue des mouvements accomplis qui ont comme résultat le maintien de leur sujet propre, de l'être vivant dans ses opérations spécifiques.

Dans un deuxième temps, je voudrais aborder la notion de fonction (ergon), liée à la notion d'acte (energeia), car il me semble que c'est autour de cette notion que l'on peut concevoir une analogie entre le fonctionnement des êtres vivants et l'ordre qui existe dans l'univers (la nature dans son entier) ou dans ses parties. Pour Aristote, les parties organiques des vivants se définissent par leurs éléments constitutifs, mais aussi et principalement par leur fonction. Et la vie est possible lorsque toutes les fonctions des organes ou des facultés se réalisent dans une certaine harmonie. Cette analyse par le moyen de la fonction est peut aussi être utilisée, même si c'est dans une mesure moins forte pour comprendre l'ordre entre les différentes parties de l'univers. Regarder l'univers ou une de ses parties comme animé d'un mouvement semblable au vivant devient alors une procédure d'explication scientifique valable, qu'Aristote choisit d'utiliser, 
plus que comme une affirmation stricte du caractère vivant du monde et de ses parties.

\section{Jean-Pierre VANANDRUEL}

[Thèse en philosophie antique - Université Paris I Panthéon-Sorbonne. - Directeur de thèse: Mme Annick Jaulin, Pr Univ. Paris I. - Laboratoire de recherche: UMR 7219, SPHERE/GRAMATA. - Sujet de thèse: "L'analyse du mouvement dans les traités de philosophie de la nature et dans les traités métaphysiques d'Aristote »].

\section{La place de l'organisme dans les dynamiques évolutives : persistance, changement, sélection}

Bien que la notion d'organisme constitue l'un des nœuds théoriques fondamentaux de l'histoire de la biologie, après le tournant de la théorie synthétique de l'évolution, elle semble avoir sombré dans l'oubli, au bénéfice de ce que Kim Sterelny et Paul Griffiths ont appelé une «vision de l'évolution du point de vue du gène " ("gene's eye view of evolution») (Sterelny et Griffiths, 1987). L'intérêt des biologistes semble donc s'être déplacé vers les espèces et, bien plus encore, vers les gènes, comme en témoigne le fait que le pilier fondamental de la théorie synthétique de l'évolution est constitué par la génétique des populations. Cette discipline, née de la "synthèse » entre la génétique mendelienne et la théorie de la sélection naturelle, consiste en l'étude de la variation des fréquences relatives des gènes et des génotypes au sein de groupes bien délimités d'organismes (les populations), ainsi que des causes de cette variation, l'une desquelles étant précisément la sélection naturelle. Dans un tel cadre, les dynamiques évolutives sont ainsi interprétées comme des mouvements inhérents à un "pool» de gènes, causés par des forces qui n'agiraient que sur ces derniers et dont les effets seraient donc mesurables à l'aide de modèles statistiques de variation des fréquences géniques.

Le but de cette intervention sera, par conséquent, d'explorer la possibilité d'une nouvelle «vision de l'évolution du point de vue de l'organisme », de sa capacité de persistance, de changement et d'adaptation à la pression de la sélection naturelle. Nous nous intéresserons tout particulièrement à l'importance épistémologique de cette notion et à son rôle explicatif, ainsi qu'à la place qu'elle occupe dans une classe de modèles mathématiques évolutifs (modèles de maximisation de la fitness) bien distincte de celle des modèles de dynamique des populations. Pour ce faire, nous mènerons une analyse à la fois conceptuelle et historique, en consacrant une attention toute particulière aux travaux pionniers, mais très différents par bien des aspects, de Ronald Fisher et de Sewall Wright.

Nicola BERTOLDI

[Après avoir obtenu une licence en philosophie à l'Université de Bologne (Italie), j'ai intégré l'École Normale Supérieure de Paris, où j'ai poursuivi mes études d'histoire et de philosophie de la biologie. Après avoir obtenu un M2 en biologie systémique et synthétique (Master AIV, Approches Interdisciplinaires du Vivant), j'ai commencé une thèse à l'Institut 
d'Histoire et de Philosophie des Sciences et des Techniques sous la direction de M. Philippe Huneman, portant sur une analyse historique et philosophique de différentes tentatives de formaliser la théorie de l'évolution].

\section{PHILOSOPHIES FÉMINISTES}

\section{Autour de l'épistémologie féministe}

Dans cet atelier, nous nous proposons de réfléchir à ce que le féminisme peut exiger d'une démarche philosophique et d'une pratique de recherche, et à ce qu'il peut apporter sur le plan méthodologique.

Nous partirons des critiques féministes qui ont été adressées aux critères épistémologiques dominants, à la prétention à l'universalisme et à l'objectivisme de la philosophie et des sciences sociales (Sandra Harding, Donna Haraway). Nous nous demanderons quelles démarches, quelles méthodes, quelles précautions et protocoles de recherche ont pu et peuvent être inventés par des chercheur.e.s féministes, pour produire un savoir philosophique critique et situé.

Nous travaillerons à partir d'un corpus de textes et d'interventions présentant des expériences de recherche personnelles pour comprendre ce que peut être une philosophie féministe, et quelles en sont les implications épistémologiques, méthodologiques et politiques.

\section{1) Axe épistémologique : la philosophie comme savoir situé}

Étant donné que la philosophie féministe de la connaissance met à mal l'universalisme des concepts, en en soulignant l'ancrage socio-historique, comment allier philosophie et études féministes? Comment mettre la philosophie en rapport avec le réel social et politique? Quelles inventions méthodologiques sont possibles?

\section{2) Axe politique : "comment s'en sortir? "(Sarah Kofman)}

Comment des outils critiques développés par certain.e.s philosophes peuvent-ils être utilisés par les personnes dans leur expérience quotidienne, et par des militant.e.s dans la construction de luttes et de solidarités? Nous pouvons prendre pour exemple deux philosophes contemporain.e.s dont le succès dans le monde militant a été et reste éclatant: Michel Foucault et Judith Butler. Que devient la philosophie lorsqu'elle se met au service d'une lutte politique? Comment certains concepts deviennent-ils des outils politiques et militants, et comment ce processus peut-il les modifier, les enrichir, leur donner un nouveau sens? 


\section{Références :}

Harding Sandra (éd.), The Feminist Standpoint Theory Reader - Intellectual and Political Controverses, Routledge, New-York 2004.

Haraway Donna, « Situated Knowledges: The Science Question in Feminism and the Privilege of Partial Perspective », Feminist Studies 14, n³, Fall 1988, p. 575-599. Trad. fr. in Manifeste cyborg et autres essais - Sciences, Fictions, Féminisme, anthologie établie par Laurence Allard, Delphine Gardey et Nathalie Magnan, Exils Éditeurs, Paris 2007.

Revue « Comment s'en sortir ? » : http://commentsensortir.org/

Mona GÉRARDIN-LAVERGE

\section{PHILOSOPHIE MORALE ET AGENTIVITÉ}

\section{Hume et la critique de la souveraineté pratique de la raison}

Les textes humiens consacrés à l'entendement sont célèbres pour avoir dégagé la composante affective de ce dernier. Cette intervention envisage ce que devient le concept de raison dans les écrits abordant les passions. Une critique de la raison s'y trouve reconduite, qui ne vise plus les revendications théoriques de l'entendement mais ses prétentions pratiques (Traité de la nature humaine, livre II, partie III, section 3 ; Dissertation sur les passions, section V). Afin de réfuter l'idée selon laquelle la raison pourrait soumettre les passions et gouverner la volonté, Hume produit deux arguments. D'une part, la raison n'est pas capable de mouvoir la volonté. D'autre part, les passions ne sont pas susceptibles de vérité ou de fausseté. Tout l'effort de l'argumentation humienne consiste ainsi à neutraliser la possibilité même d'un combat de la raison et de la passion. En affirmant que la raison ne peut pas se hisser à l'agentivité, Hume souligne en effet qu'elle ne peut pas s'opposer à la puissance motrice des passions ; en déclarant que les passions ne sont pas susceptibles de qualification épistémique, il manifeste qu'elles ne peuvent pas s'opposer à la normativité cognitive de la raison : la raison est aussi étrangère à la sphère pratique que les passions le sont à la sphère épistémique. La critique humienne de l'idée d'un combat de la raison et des passions consiste ainsi à affirmer qu'il manque aux deux protagonistes un terrain commun où se livrer combat. Si le combat est impossible, c'est parce que les prétendus adversaires ne jouent pas dans la même arène - c'est pourquoi le topos du combat de la raison et de la passion doit être remplacé par le paradigme de la raison esclave des passions, qui fixe la nature réelle de leurs rapports en matérialisant la différence de leurs statuts respectifs. 
Ceci fait, il devient visible que la critique de la raison théorique et la critique de la raison pratique obéissent chez Hume à des stratégies antirationalistes symétriques. Dans le domaine théorique, l'entendement était rapporté aux concepts de coutume, de croyance et d'instinct, opérant, pour le dire vite, une sensualisation de la raison, c'est-à-dire rapprochant la raison de ce que l'on considère habituellement comme son autre. Dans le domaine pratique, au contraire, la raison se voit caractérisée comme la pure «découverte du vrai ou du faux », creusant l'écart entre raison et affectivité, alors que Hume avait auparavant largement insisté sur leur solidarité.

Sophie BERGONT

[Sujet de thèse: "Raison et empirisme chez David Hume». - Directeur de thèse: André Charrak. - Laboratoire de rattachement : PhiCo (composante EXeCO)].

\section{Les faits et les raisons, une dichotomie?}

La conception la plus courante des faits consiste à les considérer comme des événements objectifs ayant lieu dans la réalité. Le monde est tout ce qui a lieu, l'ensemble des faits. Or une raison ne peut pas avoir le même statut ontologique. En effet, pour un agent, une raison est, sur le plan théorique, une justification de croire quelque chose, et, sur le plan pratique, une justification d'agir d'une certaine manière. Les raisons ne sont donc pas des événements, pas même des événements psychologiques. Elles sont des propositions permettant des inférences relatives à ce qui est vrai, ou à ce qui est bien. En d'autres termes, la relation de justification n'étant pas une relation réelle mais une relation logique, les raisons ne sont pas identifiables aux faits. C'est pourquoi nombre de philosophes s'inspirant de Sellars, comme McDowell, opposent diamétralement « l'espace naturel des causes » et « l'espace logique des raisons ».

Cependant, il existe une autre conception des faits, que l'on rapporte à Frege : les faits ne sont rien de plus que des "pensées vraies », ou dans des termes plus contemporains, des propositions vraies. Les faits ne sont pas des événements, ils n'ont pas lieu dans le monde. Plutôt qu'ontologique, un fait est une notion sémantique. Le but de mon intervention est de défendre cette conception des faits, et d'en montrer l'intérêt. Tout d'abord, elle implique que la dualité entre faits et raisons ne tienne plus. Car si un fait est une proposition vraie, alors un fait peut être une raison de croire tout ce qui est déductible de celui-ci. On retrouve ainsi une platitude : un fait peut être une raison de croire quelque chose, tout en échappant au fameux mythe du donné, à savoir l'erreur selon laquelle une réalité naturelle pourrait à elle seule valoir justification épistémique. Et sur le plan pratique, il en est de même: le fait qu'une chose soit bonne et le fait que nous puissions empiriquement la réaliser donnent une raison de l'accomplir. Alors qu'il aurait été étrange de soutenir qu'une réalité naturelle nous donne le devoir de 
faire quelque chose, ce que Moore épinglait sous le nom de sophisme naturaliste.

Ainsi, il n'y a de faits que pour un agent placé dans un contexte où des questions normatives se posent à lui. L'essentiel du fait n'est pas ce dont il parle, mais sa vérité, parce que c'est la vérité elle-même qui constitue une raison d'inférer telle ou telle autre chose. Toute conception séparant les causes et les raisons doit donc ramener les faits du côté des raisons, et non des causes.

Sylvain THEULLE

[Laboratoire : EXeCO. - Thèse sous la direction de Sandra Laugier. - Titre de thèse : "Les critiques de la dichotomie des faits et des valeurs »].

\section{PHILOSOPHIE DE LA PERCEPTION, ONTOLOGIE ET ESTHÉTIQUE}

\section{La distinction entre la perception et la sensation dans le toucher}

Nous allons traiter la question de la distinction entre la «perception » et la « sensation » sous l'angle de la spatialité de l'expérience perceptive. Il est souvent suggéré que les perceptions, contrairement aux sensations, concernent le monde extérieur indépendant de l'esprit et qu'elles localisent les objets dans l'espace tridimensionnel. Nous nous interrogerons donc sur la différence éventuelle entre les sensations et les perceptions tactiles. Car la spatialité du toucher semble être particulière : les stimulations sensorielles semblent être localisées à la fois sur le corps et dans le monde extérieur. Nous argumenterons que les sensations tactiles ne localisent pas nécessairement les stimuli dans le monde extérieur malgré qu'elles le font sur le corps. Nous essayerons de montrer que, d'un point de vue phénoménologique, ce qui confère à l'expérience tactile son caractère perceptif, c'est l'expérience du sujet de son propre effort physique face au monde extérieur. Celle-ci constitue la spatialité tridimensionnelle de l'expérience, comme l'indépendance de l'objet de l'expérience vis-à-vis de l'esprit.

Alireza BANI SADR

[Titre de la thèse : "La détection de l'objet de l'expérience perceptive ». - Directeur de thèse: Max Kistler. - Laboratoire : Institut d'histoire et de philosophie des sciences et des techniques (IHPST)]. 


\section{L'œil et l'esprit chez Spinoza : la peinture est-elle toujours muette?}

Spinoza est un auteur bien silencieux quand il s'agit de parler d'art. S'il lui arrive de faire référence à la musique, au théâtre ou à la danse, c'est uniquement pour les utiliser à titre d'exemples, comme pour affirmer la relativité des notions de bien et de mal (la musique bonne pour le mélancolique, mauvaise pour l'affligé, ni bonne ni mauvaise pour le sourd), ou encore pour expliquer que le corps a besoin d'une alimentation variée et qu'il est bon d'être affecté par différentes activités tels que « les parures » ou le « théâtre ». La peinture, quant à elle, n'échappe pas à la règle. Spinoza n'y fait référence que quatre fois, et elles se trouvent majoritairement dans la partie II de l'Éthique (prop. 43, 48 et 49), où Spinoza emploie toujours la même formulation, à savoir celle de la "peinture muette sur un tableau ». Cette étrange expression n'apparait donc pas n'importe où, elle est condensée à la fin de la partie II lorsqu'il est question de l'idée vraie, et plus précisément de la certitude de l'idée vraie. Spinoza refuse la positivité, ne serait-ce que méthodologique, du doute cartésien en lui opposant l'absolue certitude qui accompagne et redouble chaque idée. Avoir une idée vraie, c'est toujours, pour Spinoza, avoir l'idée de cette idée vraie, autrement dit savoir que l'on sait. À l'idée vraie définie comme « une manière de penser, à savoir le comprendre même », Spinoza oppose donc une idée considérée comme «quelque chose de muet comme une peinture sur un tableau ». Dès lors, deux problèmes méritent d'être posés : d'abord, en quoi une peinture sur un tableau est-elle une chose muette ? Et, en second lieu, la peinture estelle toujours muette?

Peut-être cet intérêt pour une peinture muette chez un auteur fort muet à son égard peut sembler suspect. Pourtant, le fait est que Spinoza a vécu aux Pays-Bas à l'époque de l'âge d'or de la peinture flamande. Avant son départ d'Amsterdam, il séjournait à quelques rues seulement des ateliers de Vermeer et de Rembrandt, dont il est fort probable qu'il connaissait les toiles. Une rumeur laisse même entendre qu'il aurait servi de modèle au Géomètre et à l'Astronome de Vermeer (tous deux élaborés en 1668). De plus, Spinoza lui-même dessinait, principalement des autoportraits. Car en effet, si la peinture sur un tableau est muette, qu'en est-il de l'activité du peintre, de cette peinture qui s'élabore au fur et à mesure des traits de pinceaux ou des coups de crayons? N'est-ce pas là l'expression d'une puissance, et a fortiori d'une puissance dont la forme pourrait s'adapter à une vie éthique et sage ? En s'appuyant sur le cas de la peinture, il conviendra d'élargir la réflexion pour interroger l'importance, voire même la prédominance, de la vision et des règles d'optique chez Spinoza, en particulier lorsqu'il est question du troisième genre de connaissance, c'est-àdire de celui-là même qui conduit l'homme à la liberté et à la béatitude. En effet, n'est-il pas possible d'analyser cette science " intuitive», celle qui s'opère «d'un seul coup d'œil », à partir de son rapport à l'optique et à 
la peinture? Pour conclure, cette intervention n'est rien d'autre qu'une invitation à parcourir ensemble les silences de Spinoza.

Nicolas BOUTELOUP

[Thèse effectuée au CHSPM/EA 1451, sous la direction de Chantal Jaquet].

\section{Le rêve et la vie : éléments d'une ontologie du surréalisme}

Le surréalisme se reconnaît à son goût cultivé du paradoxe, du non-sens, des forces obscures de la pensée, de l'arbitraire et de l'occulte. Son histoire tumultueuse, la variété des personnalités et des interprétations qui s'en sont revendiqué, au-delà de la personnalité controversée d'André Breton, tendent à ne laisser subsister que l'image d'Épinal d'une esthétique du bizarre et de l'oxymore qui peine de nos jours à véritablement surprendre. Il s'agira ici de tenter de reconstituer une unité d'inspiration permettant de caractériser le surréalisme au-delà des individualités qui s'en sont revendiquées, en dégageant la vision du monde qui l'anime. Une hypothèse historique et philosophique guide ce projet, l'idée que le surréalisme s'inscrit dans une continuité essentielle avec le projet du romantisme allemand: réconcilier, par l'activité poétique, l'être humain avec lui-même, offrir une nouvelle image du monde, où les parts subjective (notamment dans son rapport à l'imagination) et sociale (notamment politique) seraient réunies. Mais alors que le romantisme allemand s'est développé au sein d'un dialogue étroit avec la philosophie de son temps, par la lecture de Kant et de Fichte, échangeant avec des philosophes contemporains comme Schelling ou Hegel, le surréalisme s'est développé historiquement sans grand écho du côté des penseurs (quelques discussions par Camus, Benjamin ou Adorno sont à mentionner, de même que l'important ouvrage de F. Alquié), ce qui reste surprenant à l'égard d'un mouvement artistique marqué par une élaboration théorique très forte, dont les Manifestes ne représentent que la part la plus visible; l'influence, non seulement de la psychanalyse naissante, mais aussi de l'anthropologie (Lévy-Bruhl) du marxisme ou de la dialectique hégélienne (très présente chez Breton), s'y fait sentir. Au-delà de la simple recomposition d'héritages, il s'agirait d'étudier en quoi l'effort pratique et théorique des surréalistes, dans leur revalorisation de la part sauvage et nonrationnelle de la pensée, peut composer une proposition philosophique au sens fort, à travers l'anthropologie et la cosmologie qui s'en dégagent. Comment, en dernière instance, une ontologie surréaliste est-elle possible.

Louis MORELLE

[NoSoPhi/Phico. - Directeur : Laurent Jaffro]. 


\section{SUJET ET RÉALITÉ}

\section{Repenser le réalisme : quelques contributions pragmatistes (Dewey et Putnam)}

Dans cet exposé nous allons essayer d'analyser comment la pensée de certains pragmatistes (ou neopragmatistes) nous permet de nous replacer dans le débat sur le réalisme en nous plaçant au-delà, voire même en deçà, de celui-ci. La question particulière que nous allons adopter n'est pas tant celle de l'ameublement du monde mais celle de nos relations, en tant que penseurs, vers les choses externes qui constituent notre réalité. Classiquement les réalistes ont proposé des relations de type «correspondance» dans le domaine de la philosophie du langage et de l'esprit. De l'autre côté les anti-réalistes proposent des relations de type «constructiviste » ou ce qu'on peut appeler des "projections ». Les pragmatistes, entre lesquels au premier rang, Dewey, ont très bien vu que ce type de questionnement dichotomique est biaisé par avance et que nos relations sont dans une dynamique permanente. La première tâche de notre exposé sera donc de montrer en quoi ce débat est, comme Dewey le dit, « fallacieux ». Ainsi, nous aurons les moyens de voir comment on pourrait se placer au-delà de la question de l'accès au monde car en fin de compte : nous y sommes déjà. Penser qu'on peut avoir un point de vue omniscient sur les choses («God's eye view»), c'est selon ces auteurs de la mauvaise foi philosophique.

Pourtant, cela n'implique pas qu'on devrait se résigner à admettre un certain subjectivisme ou relativisme. Comme le dit très bien Putnam : «The mind and the world jointly make up the mind and the world» (H. Putnam, Reason, Truth and History, Cambridge [1981] 1995), et c'est justement ce qu'on proposera dans la deuxième partie de notre travail : une troisième voie dans le débat ou encore, une voie intermédiaire. Comment penser cette citation de Putnam où nous trouvons emmêlés la correspondance et le constructivisme? Des notions ou questionnements clés comme l'expérience perceptive ou le rôle du langage devrons être repensés pour ces propos. Dewey et ses réflexions sur l'expérience et la place de l'homme dans son environnement, ainsi que Putnam et son "réalisme naturel ou pragmatique » nous seront très utiles et nous nous inspirerons largement de leurs travaux pour montrer qu'outre une dichotomie et un débat, qui prennent la forme du dogmatisme, il existe la possibilité d'avancer réellement dans ce type de questionnements.

Aurora ALBAN

[Titre de la thèse: "Repenser le réalisme contemporain: les apports phénoménologiques et pragmatiques ", sous la direction de Jocelyn Benoist. - Laboratoire de rattachement: PhiCo]. 


\section{Être et image : une approche de la subjectivité chez Maître Eckhart}

La notion de subjectivité pose au sein du contexte de pensée de Maître Eckhart de Hochheim (théologien dominicain du tournant des $\mathrm{XIII}^{\mathrm{e}}$ et $\mathrm{XIV}^{\mathrm{e}}$ siècles) un problème de taille : en son acception «moderne »-définie par Heidegger dans Die Metaphysik als Seinsgeschichte comme rencontre des motifs de la Subjektivität et de la Subjektität (associée à l'hupokeimenon d'Aristote) -, le terme de sujet n'a de sens, pour le Thuringien, que lorsqu'il est appliqué au créateur lui-même, dans la mesure où celui-ci est, selon les termes du Commentaire au livre de l'Exode, à la fois le revendicateur exclusif du statut d'ego, et le sujet (subiectum) du monde - son support ontologique. Or, la double défense de l'idée, dans un premier temps, d'un Dieu-sujet, et, dans un second temps, d'un monde (sup)porté dans l'être par lui soulève de nombreuses questions pour l'ontologie et l'anthropologie philosophiques, au premier plan desquelles figure celle du statut de l'homme, lequel est défini par Eckhart, à la suite de Gen. 1,26, comme « image » de Dieu. En effet, le statut de l'homme se présente au sein de la pensée eckhartienne comme changeant dans la mesure où il est à la fois une créature corporelle, et donc temporelle, créée et mortelle, et un être intellectuel qui, dans les termes de la Questio utrum in deo sit idem esse et intelligere, n'a pas « la raison du créable ».

Notre contribution se donnera pour fin de se pencher sur la signification, au sein du contexte de pensée eckhartien, des termes d'être, de sujet et d'image, de manière à faire ressortir :

1. quel sens il peut y avoir à parler, chez Maître Eckhart, d'une conception de Dieu comme sujet, et qu'est-ce qui semble permettre l'émergence de ce motif conceptuel, à la lumière des sources identifiables de la pensée du Thuringien ;

2. de quelle manière le Dieu d'Eckhart est également «être» dans la mesure où de nombreux passages de son corpus associent l'être au créé et placent la nature divine au-delà de l'être, la qualifiant ici de puritas essendi (" pureté de l'être »), et là d'intellect incréé ;

3. dans la mesure où l'homme est cette image de Dieu qui, selon Eckhart, est capax dei (« capable de Dieu»), pouvant s'assimiler, s'unir de manière immédiate à son créateur par la pratique de l'éthique du détachement, quelle place est réservée à l'individu au sein de cette pensée de la mort de soi pour laquelle le bonheur suprême est accessible à l'homme sur terre par cette voie ?

Pierre-Luc DESJARDINS

[Co-directeurs : David Piché (UdeM) et Jean-Baptiste Brenet (Paris I). - Affilié à GRAMATA. - Titre de la thèse : "Sous-jacence, inhérence et subjectivité : approches de la notion de sujet chez Maître Eckhart ». La thèse se propose une archéologie de la notion de sujet chez Maître Eckhart]. 


\section{Inhérence, immanence, ipséité : Heidegger, lecteur des scolastiques}

Notre travail porte sur le «premier tournant» de la philosophie heideggérienne. Par ce terme, nous désignons le changement de perspective opéré par Heidegger entre la publication de sa thèse d'habilitation, le Traité des catégories et de la signification chez Duns Scot, en 1916, et celle d'Être et Temps en 1927. Nous étudions la façon dont sa conception de l'intériorité $\mathrm{du}$ «sujet» évolue, durant ces années, avec sa lecture des philosophes scolastiques. Ainsi, en 1916, Heidegger, lisant Duns Scot à partir de Husserl, refuse de considérer le sujet pensant comme le sujet d'inhérence des significations. Celles-ci sont certes dans l'âme, in anima, mais le in n'exprime pas l'inhérence (l'«être dans un sujet» aristotélicien, en hupokeimenôi einai), il réinvestit l'idée husserlienne du sens noématique, comme étant « inséparable de la conscience sans pour autant s'y trouver réellement contenu [nicht reel in ihm enthalten]» (Traité des catégories et de la signification chez Duns Scot, traduction par F. Gaboriau, Paris, Gallimard, 1970, p. 105; Gesamtausgabe 1, Frankfurt am Main, Klostermann, 1978, p. 277). Un tournant intervient cependant dans les années suivantes : la pensée du Dasein exige non seulement de rompre avec la notion d'inhérence, mais également avec l'idée d'une pensée immanente, présente chez Husserl. Dans l'Introduction à la recherche phénoménologique (cours du semestre d'hiver 1923-1924), Heidegger déconstruit ainsi la notion de "région-conscience », en exposant l'origine thomasienne des conceptions cartésienne et husserlienne du sujet. En 1916 et en 1923-24, donc, Heidegger rejette deux figures de l'intériorité (l'inhérence et l'immanence), en engageant à chaque fois un dialogue avec la philosophie scolastique. « Sujet» sans inhérence et sans immanence, le Dasein de 1927 est donc dépourvu d'intériorité au sens traditionnel du terme. On peut cependant identifier des «résidus » ou «reliquats 》 d'intériorité «dans » le Dasein de 1927 : la réflexion, réinterprétée comme réfléchissement de soi (de l'ipséité) dans le monde, l'isolement de l'être-au-monde dans l'instant de l'angoisse, ainsi que la totalité ou fermeture du Dasein qui se manifeste dans l'être-pour-la-mort. Ces « résidus » rendent possible, en 1927, une nouvelle pensée de l'intériorité, conforme à l'être du Dasein compris comme être-aumonde (In-der-Welt-sein).

Mathieu EYCHENIÉ

[Sujet de thèse: "La persistance du sujet dans la philosophie du premier Heidegger et ses sources médiévales" " - Directeur de thèse: Jean-Baptiste Brenet (Paris I); co-directeur: Alain de Libera (Collège de France). - Laboratoire de rattachement: Sphère UMR 7219 (centre GRAMATA). - Doctorant contractuel de première année]. 


\section{Qu'est-ce qu'un champ? Aspects ontologiques de l'électromagnétisme de Faraday}

On doit pour une grande part la théorie de l'électromagnétisme classique au scientifique britannique du XIX ${ }^{\mathrm{e}}$ siècle Michael Faraday. Des années 1820 aux années 1850 , il a élaboré une théorie des phénomènes électriques et magnétiques reposant sur la notion de champ, dont il est l'auteur. Cette théorie s'opposait aux théories continentales, telles celles de Coulomb ou d'Ampère, qui suivaient un paradigme newtonien et ne faisaient intervenir que des particules, des fluides et des forces rectilignes agissant à distance selon des lois en carré inverse.

Nous préciserons d'abord pourquoi l'on peut considérer que la théorie du champ de Faraday s'impose contre les théories continentales. La raison en est notamment que ces dernières ne semblent pas permettre, au contraire de la théorie du champ électromagnétique, de rendre compte adéquatement de la différence, établie par Faraday, entre deux types de réaction des corps à l'action magnétique, le paramagnétisme et le diamagnétisme.

Ensuite, nous nous demanderons quelle est l'interprétation ontologique correcte de la théorie de Faraday. Nous verrons que la signification physique que la notion de champ possède aux yeux de Faraday n'est pas celle que ses exégètes lui attribuent le plus souvent, dans la mesure où la notion de «lignes de force », très importante au demeurant dans ses écrits, ne permet pas tout à fait d'en rendre compte. Nous pensons que les lignes de force ne sont qu'un moyen de représentation graphique de l'action électromagnétique, non sa nature physique. Il faut plutôt voir dans le champ électromagnétique un déploiement dans l'espace et dans le temps d'une condition particulière que Faraday nomme "état électrotonique ». S'il est d'abord attribué par Faraday aux particules matérielles, cet état en vient au fur et à mesure des progrès expérimentaux du scientifique à être attribué à l'espace vide.

C'est pourquoi nous verrons enfin que cette conception du champ électromagnétique implique une métaphysique particulière, qui conteste la substantialité de la matière et de la force, et attribue à l'espace lui-même des propriétés qui lui font perdre, dès avant la relativité einsteinienne, son statut traditionnel. Cette métaphysique va en fait jusqu'à considérer que les entités fondamentales du monde physique ne sont que des tensions de l'espace luimême.

Kévin CAPELLI

[Sujet de recherche: "La notion de champ dans l'électromagnétisme classique, sa signification physique, son statut épistémologique et sa portée métaphysique ». - Directeur de recherche : Maximilian Kistler. - Laboratoire de rattachement : IHPST]. 